\title{
Labour Law of E.U. About the Free Circolation: a Comment on Recent Discussion According to Most Relevant Pronunciations of Justice Court
}

\author{
Antonio Vito Pasquale Boccia \\ PHD Industrial Relations - University "Alma mater studiorum" of Bologna (Italy) \\ Guest Professor at Faculty Fastip - "Aleksandër Moisiu" Universiteti i Durrësit (Albania) \\ C.M. (Fellow) at Faculty of Law - University "Ippocratica Civitas" of Salerno (Italy) \\ prof.antonioboccia@gmail.com
}

\begin{abstract}
Today, into Shengen's area, some of the european countries -among which the Austrian Republic- because of illegal immigration and because of the terrorist attacks too, decide for the temporany national frontier closing: directly between the same countries that build up the European Union. If it happens, in this period, it would mean also the unavoidable restriction of the working rights among the european citizens. But what did represent and does represent the freedom of circulation among the workers in the law Community system? Naturally there will be a law discussion about: in this occasion we try to give an aswer to this question, according to the right, but -above all- let's try to bring back the discussion on a line of law correctness, according to the recent decisions of the European Court of Justice that set limits to the topic.
\end{abstract}

Keywords: Labour Law of E.U., Free Circolation, Justice Court

\section{Il principio di libera circolazione delle persone}

Nel diritto comunitario, com'è noto, le normative che regolano la circolazione delle persone all'interno del territorio dell' Unione Europea costituiscono uno dei capitoli più rilevanti e significativi.

La libera circolazione dei cittadini della U.E. ha una sua propria caratterizzazione, prettamente economica, che tuttavia pare essere funzionale soprattutto al raggiungimento degli obiettivi europei in materia di politica sociale. Invero il diritto alla libera circolazione (dei lavoratori) è finalizzato alla costituzione di un unico mercato del lavoro su scala europea: per tale motivo a tutte le persone che lavorano all'interno del'Unione, proprio in quanto soggetti economici, deve essere assicurata la piena libertà di spostamento tra i vari Stati-membro che costituiscono la Comunità: siano essi lavoratori subordinati, lavoratori autonomi o persone giuridiche (1).

In particolare, sul punto, si è osservato che la libertà dei lavoratori ha implicato la abolizione di qualsivoglia forma di discriminazione $a b$ origine basata sulla nazionalità delle persone, con riguardo al diritto di ingresso nel territorio comunitario, all'accesso al lavoro, alle condizioni, al soggiorno ed al diritto di mantenervi la propria residenza (2).

Per l'effetto, quindi, l'esigenza di favorire la mobilità intra-comunitaria dei lavoratori ha superato i criteri (obsoleti) basati sulla nazionalità, interni ai sigoli Stati-membro: non a caso si sono succedute nel tempo una serie di norme di attuazione, a partire dal 1961, e la stessa Corte di Giustizia ha avuto modo di pronunciarsi più volte sull'argomento. (3)

Nel campo di applicazione delle normative sulla libertà di circolazione rientrano anche i componenti della famiglia del lavoratore (il coniuge e i discendenti che siano minori di anni ventuno): tuttavia c'è da dire che il diritto di soggiorno dei familiari non costituisce un diritto autonomo, bensì è naturalmente collegato alla circostanza che il lavoratore abbia già esercitato il suo proprio diritto di libera circolazione e che disponga di un alloggio. (4)

In patica l'esistenza di un mercato del lavoro comunitario permette che lavoratori e datori di lavoro possano scambiare in piena libertà le domande e le offerte di impiego, dando esecuzione ai contratti di lavoro conclusi. (5) 
Quanto ai contenuti del diritto medesimo, esso si estrinseca innanzitutto nella parità di accesso ai posti di lavoro disponibili in ciascuno dei Paesi-membro della UE, ed è identificabile nella garanzia della parità di trattamento nell'accesso all'impiego tra lavoratori nazionali e lavoratori che provengono da altri stati comunitari. (6)

In buona sostanza la garanzia di parità di trattamento trova fondamento nella impossibilità di far dipendere la assunzione del lavoratore a criteri discriminatori in ragione della sua nazionalità. (7)

In effetti il divieto di discriminazione, a contrario, rappresenta un autentico limite giuridico sia per i comportamenti dei poteri pubblici, sia per la autonomia dei privati: sicchè le clausole discriminatorie che siano contenute in norme, o contratti (individuali e/o collettivi), sono da considerarsi radicalmente nulle. (8)

Ovviamente la libera circolazione può essere parzialmente limitata dalla (legittima) richiesta di attestati di qualificazione professionale, poiché le regole per il rilascio di tali attestazioni risultano ancora essere diverse nei vari Stati-membro, in prevalenza per ciò che riguarda i lavoratori autonomi. (9)

\section{La libera circolazione dei lavoratori}

Sempre in ordine al contenuto del diritto in esame e sulla base del diritto alla libertà di circolazione di chi lavora, il principio di parità di trattamento del lavoratore costituisce, evidentemente, una parte integrante del diritto di libera circolazione: trattasi di una garanzia di carattere generale - la quale inerisce alle condizioni di lavoro e che deve trovare puntuale applicazione in relazione a tutta la materia lavoristica - su cui, peraltro, si è più volte soffermata la Corte di Giustizia. (10)

II principio di parità gode comunque di un'ampia valenza protettiva: non a caso tale garanzia è funzionale sia alla integrazione dei lavoratori migranti che alla tutela degli stessi cittadini del paese di accoglienza. (11)

Ciò detto, si osserva quanto segue: il principio di libera circolazione non può che implicare il diritto a spostarsi liberamente nel territorio degli stati-membro e, quindi, il diritto del lavoratore a lasciare il proprio territorio nazionale, onde accedere ad una attività lavorativa in un altro paese comunitario. Quanto alle formalità, esso sarà applicabile semplicemente con la presentazione di un documento di identità, senza il rilascio di alcun visto di uscita: detta facoltà coincide con il diritto di ingresso del lavoratore migrante in ognuno dei paesi della UE, che non è condizionabile da alcuna forma di visto di ingresso. (12)

Altresì, la libertà di circolazione del lavoratore si concretizza nel diritto di soggiornare senza il rilascio di alcun permesso costitutivo del diritto. (13) Accanto a tale diritto esiste la ulteriore facoltà, esercitabile dopo la cessazione dell'attività lavorativa, di continuare a risiedere sul territorio dello stato ove è stata esercitata l' attività lavorativa, sempre ricorrendo determinate condizioni di durata del lavoro. (14)

Infine, quanto alle residue limitazioni legali che sono, ad oggi, ancora poste alla libertà di circolazione dei lavoratori, giova ricordare che sussistono ancora due limiti: un primo, di carattere meramente residuale, che riguarda l'accesso dei lavoratori extra-nazionali all'impiego pubblico nelle amministrazioni dei singoli stati-membro (limite che, occorre dire, si va riducendo sempre più, sia grazie alle direttive di coordinamento in materia, sia alla luce delle numerose pronunce della Corte).

Ovviamente sussiste anche un altro -ed ulteriore- limite: il quale concerne, invece, le ragioni di ordine pubblico, di pubblica sicurezza, ovvero di sanità pubblica, su cui conviene soffermarsi alla fine del presente articolo.

Dunque è facile notare che, quanto all'oggetto del divieto, esso non può che riguardare i singoli provvedimenti, adottati in casi eccezionali da uno (o più) stati-membro, relativamente al limite di ingresso sul territorio nazionale, o alla espulsione di soggetti dal territorio medesimo (16).

Ovviamente non possono sussistere mere ragioni di carattere economico, ma solo motivazioni gravi che riguardino minacce all'ordine pubblico, alla sicurezza, o per la sanità e l'igiene: anzi, a tal proposito, l'Unione sta cercando di armonizzare i vari criteri nazionali, ancora oggi non uniformi, pur restando fermo - in capo alle competenti autorità nazionali - un certo potere discrezionale, con riferimento, in particolare, alla procedura di espulsione. (17) 


\section{La libera circolazione di servizi}

A completamento della materia che ci occupa, si deve sottolineare che il Trattato sul funzionamento della Unione, nell'evidente intento di assicurare la piena mobilità dei fattori produttivi in senso ampio, con gli articoli 56-62 TCE prevede, inoltre, la libera prestazione e circolazione dei servizi: questa costituisce, in un certo senso, il pieno completamento del diritto alla libera circolazione dei lavoratori.

In effetti per "servizi" devono intendersi, secondo l'art. 57 TFUE, «le prestazioni fornite normalmente dietro retribuzione, in quanto non siano regolate dalle disposizioni relative alla libera circolazione delle merci, dei capitali e delle persone». Tali prestazioni comprendono attività di carattere industriale, commerciale, artigiane e, infine, le libere professioni. (18)

L'art. 56 TFUE, rispetto all'esercizio dei servizi, peraltro, prevede il divieto di restrizioni nei confronti dei cittadini degli Stati membri stabiliti in un paese della Unione che non sia quello del destinatario della prestazione.

In secondo luogo, con l'art. 57 paragr. 2 TFUE, è previsto che per il prestatore (il quale, a titolo temporaneo, eserciti la propria attività in un paese diverso da quello di origine) il pieno diritto di esercitare la propria attività «alle stesse condizioni imposte da tale Stato ai propri cittadini». (20)

La libera prestazione dei servizi non può che comporsi - analogamente alla libera circolazione dei lavoratori subordinati e al diritto di stabilimento - sia del diritto di accesso all'attività che del diritto al trattamento nazionale: ma, mentre il primo diritto presuppone l'esercizio continuo e permanente di un attività in un altro Stato membro, la libera prestazione dei servizi riguarda anche un esercizio solo temporaneo e occasionale di un'attività non salariata (all'interno di ognuno degli Statimembro).

Come per il diritto al libero stabilimento, anche nella libera prestazione dei servizi sono in primo luogo vietate le discriminazioni "dirette", ossia quei casi in cui la normativa nazionale prevede espressamente un trattamento diverso e meno favorevole per i liberi prestatori rispetto a quello applicabile ai soggetti stabiliti (come nel caso della norma francese, che vietava ai soli medici stabiliti in altri Stati di visitare più di un paziente per un periodo complessivo di due giorni).

Naturalmente è fatto divieto anche delle discriminazioni "indirette" (dette anche "occulte"): ossia, per meglio dire, è vietata qualsiasi forma di discriminazione dissimulata che, sebbene basata su criteri in apparenza neutri, nella vita pratica vada a produrre lo stesso identico risultato discriminante.

Il principio è stato affermato, ad esempio con riferimento alla normativa italiana, in materia di concessione di lavori pubblici che, per quanto riguarda i subappalti, accordava la preferenza alle imprese che svolgevano la loro attività prevalentemente nel territorio della Regione interessata dai lavori (21).

Sono, infine, vietate le discriminazioni "materiali", cioè quelle che derivano dall'assimilazione della situazione del prestatore di servizi straniero a quella del prestatore nazionale rispetto a requisiti che risultano per il cittadino di uno Stato membro più difficili da acquisire (come nel caso delle normative professionali, in cui lo Stato, imponendo ai liberi prestatori la risposta a requisiti previsti dalla normativa nazionale, non tiene conto del fatto che tali soggetti sono già tenuti a rispettare i requisiti richiesti per l'esercizio dell'attività nello Stato di stabilimento) .

Si noti che, come già osservato in tema di diritto di stabilimento, mediante una giurisprudenza ormai consolidata la Corte ha assunto -nei confronti delle discriminazioni indirette o materiali alla libera prestazione dei servizi- un approccio diverso, che non si limita ad accertare l'esistenza di una discriminazione, quanto piuttosto verifica se sussiste un ostacolo alla libera circolazione dei servizi (22).

Sicchè il principio del trattamento nazionale, che è stato sancito all'art. 57 , terzo comma TFUE, non può e non deve essere inteso nel senso restrittivo della necessità della applicazione integrale della disciplina nazionale alle attività di carattere temporaneo, che siano esercitate da imprese stabilite in altri Stati: invero, piuttosto, la libera prestazione dei servizi, come del resto quella dei lavoratori -in quanto principio fondamentale sancito dal Trattato- potrà essere limitata sempre e solo da normative di carattere temporaneo che siano giustificate dal pubblico interesse e che siano rese obbligatorie per tutte le persone e le imprese che esercitano la propria attività sul territorio di tale Stato. 


\section{Indicazioni Bibliografiche}

(1) Sul punto:

M. ROCCELLA -T. TREU, Diritto del lavoro della Comunità esuropea, pp. 59 - 111, Padova, Edizioni Cedam, 2012

G. FONTANA, La libertà sindacale in Italia e in Europa. Dai principi ai conflitti, in Rassegna di diritto pubblico europeo, 2010, n. 2, pp. 97-172.

A. DI PASCALE, I diritti sociali nella giurisprudenza della Corte di giustizia dell'Unione europea: diritti fondamentali?, in Rivista di diritto internazionale, 2014, pp. 1148- 1174

(2) P. ALSTON, Labour rights and human rights, pp. 11-14, Oxford, Oxford University Press, 2005

V. HATZOPOULOS, A (more) social Europe: A political crossroad or a legal one-way? Dialogues between Luxembourg and Lisbon, in Common Market Law Review, 2005, pp. 1599-1635.

(3) Sul punto:

AGUILAR-GNZALVEZ M. C., Fondamenti e proiezione della contrattazione collettiva europea, in Diritti lavori mercati, 2013 , pp. 169-186;

B. CONFORTI, Diritto internazionale privato e della Comunità Europea, Torino, Editoriale Scientifica, 2010, pp. 4-11

L. CALAFÀ, I confini sociali dell'immigration policy dell'Unione europea, in Rivista giuridica del lavoro e della previdenza sociale, 2013, pp. 347-370.

(4) M. FALCONE, Previdenza e vantaggi sociali per i familiari dei lavoratori comunitari migranti e frontalieri secondo la Corte di giustizia, in Studi sullintegrazione europea, 2009, pp. 681-715.

S. GIUBBONI, Libera circolazione delle persone, prestazioni familiari e regole comunitarie anticumulo, in Rivista italiana di diritto del lavoro, 2010, p. II, pp. 486-492.

(5) L. NOGLER, Diritto del lavoro e diritto contrattuale europeo: un confronto non più rinviabile, in Giornale di diritto del lavoro e di relazioni industriali, 2009, pp. 171-205.

A. COSTA, M. CICCIÙ, Distacco dei lavoratori in ambito Ue. Istruzioni dopo l'entrata in vigore, dal $1^{\circ}$ maggio 2010, dei regolamenti in materia di sicurezza sociale, in Guida al Lavoro, 2010,

n. 28, pp. I-XXXVI (inserto).

(6) M.COUSINS, Free Movement of Workers, EU Citizenship and Access to Social Advantages, in Maastricht Journal, 2007, n. 4, pp. 343-360.

(7) O. GOLYNKER, Student loans: the European concept of social justice according to Bidar, in European law review, 2006, pp. 390-401.

E. NALIN, Cittadinanza dell'UE, libera circolazione e diritto di stabilimento dei lavoratori transfrontalieri, in Sud in Europa, 2008, n. 10, pp. 13-14.

(8) Sul punto:

S. O'LEARY, Equal treatment and EU citizens: A new chapter on cross-border educational mobility and access to student financial assistance, in European Law Review, 2009, pp. 612-627.

D. MARTIN, La libre circulation des personnes : au-delà de l'évolution et révolutions, la perpétuelle quête de sens, in Revue des Affaires Européennes, 2012, pp. 85- 97. 
A. CELOTTO, Quando un «diritto» non è un diritto: sulla circolazione e soggiorno dei cittadini comunitari, in Quaderni Costituzionali, 2010, pp. 859-860.

(9) M. FUCHS, The Bottom Line of European Labour Law, in The International Journal of Comparative Labour Law and Industrial Relations, 2004, n. 3, pp. 423-444.

D. GOTTARDI, Diritti sindacali e libertà economiche al Parlamento europeo. I nodi del coordinamento sistematico, in Lavoro e diritto, 2008, pp. 555-578.

(10) P. MATTERA, Politique sociale (le rapport 2010 de la Commission européenne sur la protection et l'inclusion sociale), in Les dossiers européens: actualités en bref, in Revue du Droit de l'Union européenne, 2010, n.1, pp.119-120

(11) U. VILLANOVA, Modalità operative per l'ingresso dei lavoratori neocomunitari, in Guida al Lavoro, 2004, n. 20, pp. 12-16.

M.C. BARUFFI, I diritti sociali nell'Unione Europea dopo il Trattato di Lisbona, in Guida al Lavoro, 2010, n. 5, pp. 44-49.

(12) B. GASPARRO, La libera circolazione dei lavoratori europei dopo il regolamento 492/2011 -Freedom of movement of European workers after the european regulation no. 292/2011, in Massimario di Giurisprudenza del Lavoro, 2011, pp. 823-839.

(13) P. PICIOCCHI, I recenti orientamenti della Corte di Giustizia in materia di politiche sociali, in Diritto pubblico comparato ed europeo, 2011, pp. 142-167.

A. CIARINI, Lavoro e attivazione in Europa. Investimento sociale o creazione diretta di nuova occupazione?, in Quaderni rassegna sindacale, n. 1, 2013, pp. $143-161$.

A. MONTANARI, Diritto internazionale privato del lavoro e ordinamento comunitario, in Massimario di Giurisprudenza del Lavoro, 2010, pp. 153-164.

(14) G. CAGGIANO, II bilanciamento tra libertà di circolazione dei fattori produttivi ed esigenze imperative degli stati membri nel mercato interno, in Studi sull'integrazione europea, 2012,pp. 295-327.

(15) B. NASCIMBENE, La centralità della persona e la tutela dei suoi diritti, in Studi sullintegrazione europea, n.1, 2013, pp. $9-18$

(16) L. IDOT, D. SIMON, A. RIGAUX, Libre circulation des travailleurs, in Europe, genn. 2011, p. 24.

(17) I. VIARENGO, Costituzione europea e politica sociale comunitaria, in Guida al lavoro, 17(2005), pp. 28-35.

A. BAYLOS GRAU, Crisi, modello europeo e riforma del lavoro, in Lavoro e diritto, 2010, pp. 473-489.

(18) L.ZOPPOLI, Unione Europea e lavoro sommerso: nuove attenzioni e vecchie contraddizioni, in Rivista giuridica del lavoro e della previdenza sociale, 2008, n. 1, pp. 81-106.

(19) M. ROCELLA, Formazione, occupabilità, occupazione nell'Europa comunitaria, in Giornale di diritto del lavoro e di relazioni industriali, 2007, n. 1, pp. 187-236.

(20) G. FALASCA, Unione europea: approvato il codice della libera circolazione dei lavoratori, in Guida al lavoro, n. 26, 2011, pp. 42 - 43.

(21) A. CANEPA, Spazio europeo della ricerca e «permesso di soggiorno scientifico». La procedura d'ingresso per ricercatori extra-comunitari tra disciplina europea e attuazione nazionale, in Rivista Italiana di Diritto Pubblico Comunitario, 2010, pp. 1183-1218

(22) Sul punto:

G. PRIOLA, Lavoro e Costituzione Europea, in ADL, 2004, pp. 519-529.

A. PERULLI, Diritti sociali fondamentali e regolazione del mercato nell'azione esterna dell'Unione europea, in Rivista giuridica del lavoro e della previdenza sociale, 2013, pp. 321-345. 
M. IUS, Il principio di proporzionalità delle misure restrittive di uno Stato membro rispetto al diritto di libera circolazione garantito dall'Unione, in Lo stato civile italiano, n. 3, 2011, pp. 23 - 27.

\section{COMMENTI SULLA GIURISPRUDENZA DELLA CORTE EUROPEA DI GIUSTIZIA}

E.ADOBADI (a cura di), La Corte di Giustizia interpreta la direttiva n. 2004/38/Ce sul diritto dei cittadini dell'Unione di circolare e di soggiornare all'interno degli Stati membri, in II Massimario annotato, in Diritto comunitario e degli scambi internazionali, n. 2, 2011, pp. 289 - 307.

Nota a sentenza della Corte del 5 maggio 2011, causa C-434/09, Shirley McCarthy c. Secretary of State for the Home Department

A. BOUVERESSE, Circulation des Travailleurs, Notion de travailleur, in L. Idot, D. Simon, A. Rigaux, Marché interieur, in Europe, n. 4, 2013, pp. 25- 26.

Nota a sentenza della Corte di Giustizia, del 21 febbraio 2013, Causa C-46/12, L. N. c. Styrelsen for Videregående Uddannelser og Uddannelsesstøtte.

A. BOUVERESSE, Circulation des Travailleurs, Condition de résidence, in L. Idot, D.Simon. A. Rigaux, Marché Intrérieur, in Europe, n.2, 2013, p. 30. Nota a sentenza della Corte di Giustizia, del 13 dicembre 2012, Causa C-379/11, Caves Krier Frères Sàrl c. Directeur de l'Administration de l'emploi.

V. CAPUANO, La libera circolazione dei calciatori nell'Unione europea tra vecchie questioni e nuovi scenari: il caso Bernard, in Rivista italiana di diritto del lavoro, n. 1, 2011, pp. 189 - 196.

Nota a sentenza della Corte (grande sezione) del 16 marzo 2011, causa C-325/08, Olympque Lyonnais SASP c. Olivier Bernard e Newcastle United UFC.

E. CHITI, S. SCREPANTI (a cura di), Corte di Giustizia e Tribunale dell'Unione europea, in Giornale di diritto amministrativo, 2012, pp. 648-649.

Contiene:Unione europea: Politica sociale, p. 648.

Nota a sentenza della Corte di Giustizia del 24 aprile 2012, Causa C-571/10, Servet Kamberaj c. Istituto per l'Edilizia sociale della Provincia autonoma di Bolzano (IPES) e altri

R. CONTI, R. FOGLIA (a cura di), Lettori di lingua straniera e discriminazioni in base alla cittadinanza, in Osservatorio della Corte di Giustizia CE, in Il Corriere giuridico, 2008, pp. 1168-1169. Nota a sentenza della Corte di giustizia del 15 maggio 2008, causa C-276/07.

A. CORRADO, Discriminanti i requisiti degli studi "interni" e del genitore migrante residente nel

Paese, in Guida al Diritto, 2005, n. 39, pp. 115 e ss.Nota a sentenza della Corte di giustizia del 15 Settembre 2005, causa C-258/04

L. DRIGUEZ, Circulation des travailleurs, Regle nationale anti-cumul appliquee a une pension de survie, in L. IDOT, D. SIMON, A. RIGAUX, Marche interieur, inEurope, n.5, 2013, pp. 22-23. Nota a sentenza della Corte di Giustizia, del 7 marzo 2013, Causa C-127/11, Aldegonda van den Booren c. Rijksdienst voor Pensioenen.

L. DRIGUEZ, Circulation des travailleurs. Sécurité sociale, totalisation des périodes d'assurance et

égalité de traitement, in Europe, n. 2, 2012 , p. 25. Nota a sentenza della Corte del 15 dicembre 2011, causa C257/10, Försäkringskassan c. Elisabeth Bergström.

L. DRIGUEZ, Securite sociale, Totalisation des periodes d'assurance et ajustement des modes de calcul des pensions theoriques, in L. Idot, D. Simon, L. Driguez,Marche interieur, in Europe, n. 4, 2013, p. 27. Nota a sentenza della Corte di Giustizia, del 21 febbraio 2013, Causa C-282/11, Concepción Salgado González c. Instituto Nacional de la Seguridad Social (INSS) e Tesorería General de la Seguridad Social. 
L. DRIGUEZ, Securite sociale, Droit aux prestations familiales pour orphelins, in L. Idot, D. Simon, L. Driguez, Marche interieur, in Europe, n. 4, 2013, pp. 26-27.Nota a sentenza della Corte di Giustizia, del 21 febbraio 2013, Causa C619/11, Patricia Dumont de Chassart c. Office national d'allocations familiales pour travailleurs salariés.

S. GROSBON, Libre circulation et systèmes de sélection universitaire : une équation complexe, in Revue des Affaires Européennes, n. 3, 2009 - 2010, pp. 619 - 626. Contiene: Recoinnaissance mutuelle des diplômes : prise en compte de l'expérience pratique, p. 24. Nota a sentenza della Corte del 2 dicembre 2010, cause C-422, 425 e 426/09, Vandorou, Giankoulis et Askoxilakis.

A.MAFFEO, Ai fini della qualifica "lavoratore" il carattere reale ed effettivo della prestazione prevale sulle motivazioni personali che hanno indotto il prestatore ad entrare nell Stato membro, in Diritto pubblico comparato ed europeo, 2013,pp. 703-707. Nota a sentenza della Corte di Giustizia,21 febbraio 2013, causa C-46 /12,L. N. C. Styrelsen for Videregaende Uddannelser og Uddannelsesstotte.

\section{MICHAEL, Circulation des Travailleurs, in L. Idot, D. Simon, A. Rigaux, Marché interieur, in}

Europe, n. 4, 2013, pp. 24- 25.Nota a sentenza della Corte di Giustizia, del 28 febbraio 2013, Causa C-544/11, Petersen et Petersen.

V. MICHEL, Circulation des Travailleurs, in L .Idot, D. Simon, A. Rigaux, Marché intérieur, in Europe, n.1, 2013, pp. 3132. Nota a sentenza della Corte di Giustizia, dell'8 novembre 2012, causa C-268/11, Atilla Gülbahce c. Freie und Hansestadt Hamburg.

V. MICHEL, Circulation des travailleurs. Accord d'association UE-Turquie et double nationalité, in Europe, 2012, pp. 2526. Nota a sentenza della Corte di giustizia del 29 marzo 2012, Cause riunite C-7/10 e C-9/10, Staatssecretaris van Justitie contro Tayfun Kahveci (C-7/10) e Osman Inan (C-9/10).

J. PERTEK, Reconnaissance mutuelle des diplomes d'architecte, in Revue des affaires européennes,

2014, pp. 441- 444 ota a sentenza della Corte di giustizia del 30 aprile 2014, C-365/13, Ordre des architectes/ ètat BelgeBelgique.

F. SCIOTTO, Giocatori «promessa» e libera circolazione dei calciatori professionisti: la Corte di giustizia europea riconosce un indennizzo per la formazione, in Rivista italiana di diritto del lavoro, n. 1, 2011, pp. 197 - 201. ota a sentenza della Corte (grande sezione) del 16 marzo 2011, causa C-325/08, Olympque Lyonnais SASP c. Olivier Bernard e Newcastle United UFC. 